\title{
Imagens multipolarizadas do sensor Palsar/Alos na discriminação das fases fenológicas da cana-de-açúcar
}

\author{
Michelle Cristina Araujo Picoli(1), Rubens Augusto Lamparelli(2), Edson Eyji Sano ${ }^{(3)}$ e Jansle Vieira Rocha(1) \\ (1)Universidade Estadual de Campinas (Unicamp), Faculdade de Engenharia Agrícola, Avenida Cândido Rondon, no 501, \\ CEP 13083-875 Campinas, SP. E-mail: michelle.picoli@feagri.unicamp.br, jansle.rocha@feagri.unicamp.br (2)Unicamp, Núcleo Interdisciplinar \\ de Planejamento Energético, Campus Unicamp, CEP 13083-896 Campinas, SP. E-mail: rubens@nipeunicamp.org.br ${ }^{(3)}$ Embrapa Cerrados, \\ BR-020, Km 18, CEP 73301-970 Planaltina, DF. E-mail: edson.sano@embrapa.br
}

Resumo - O objetivo deste trabalho foi avaliar o potencial das imagens multipolarizadas do sensor-radar Palsar/Alos em diferenciar as fases fenológicas da cana-de-açúcar. Valores digitais de quatro imagens do sensor, dos meses de fevereiro, maio, agosto e outubro de 2008, com polarizações HH (emissão e recebimento de onda na polarização horizontal) e HV (emissão de onda na polarização horizontal e recebimento na vertical), foram convertidos para coeficientes de retroespalhamento $\left(\sigma^{\circ}\right)$, para a análise de dados de cana-de-açúcar, cultivadas em talhões na região nordeste do Estado de São Paulo. Foram selecionadas três variedades, em diferentes estágios fenológicos: RB85-5156, seis talhões; RB86-7515, dez talhões; e RB92-5345, dez talhões. As diferenças entre as fases fenológicas foram avaliadas para cada uma das variedades e, também, entre as variedades. A utilização simultânea ou não dos dados do sensor Palsar/Alos, obtidos em duas polarizações, foi capaz de discriminar as diferentes fases de crescimento da cana-de-açúcar, com exceção da fase de crescimento dos colmos e a fase de maturação, em que não foi observada diferença significativa.

Termos para indexação: Saccharum, fase de crescimento, radar de abertura sintética, retroespalhamento, SAR.

\section{Multipolarized Palsar/Alos images to discriminate sugarcane phenological phases}

\begin{abstract}
The objective of this work was to evaluate the potential of multipolarized Palsar/Alos satellite images to discriminate phenological phases of sugarcane. Digital values from four digital images of February, May, August, and October 2008, with HH (sending and receiving wave in horizontal polarization) and HV polarizations (sending wave in horizontal polarization and receiving in vertical polarization), were converted to backscattering coefficients $\left(\sigma^{\circ}\right)$ for data analysis of sugarcane cultivated in the northeastern of the state of São Paulo, Brazil. Three varieties were selected at different phenological stages: RB85-5156, six stands; RB86-7515, ten stands; and RB92-5345, ten stands. The differences between the phenological phases were analyzed for each variety, and also between varieties. The single or dual-poralized Palsar/Alos data, obtained in two polarizations, were able of discriminating the different phases of sugarcane growth, except for the grand growth period and maturity phase, in which no significant difference was observed.
\end{abstract}

Index terms: Saccharum, growth phases, synthetic aperture radar, backscatter, SAR.

\section{Introdução}

A maioria das pesquisas realizadas até o momento para compreender o comportamento espectral da cana-de-açúcar vem utilizando dados multiespectrais e hiperespectrais na faixa óptica do espectro eletromagnético (Abdel-Rahman \& Ahmed, 2008; El Hajj et al., 2009; Lebourgeois et al., 2010; Vieira et al., 2012). No Estado de São Paulo, os meses de janeiro e fevereiro são os mais favoráveis para a coleta de dados de sensoriamento remoto sobre cana-de-açúcar (Picoli et al., 2009; Rudorff et al., 2010). Fevereiro é o melhor mês para relacionar a resposta espectral da cana-de-açúcar com a massa de colmos, que está diretamente ligada à produtividade da planta. Porém, esse mês também é caracterizado por elevada precipitação pluvial. A precipitação pluvial média de 30 anos (1971-2000) em São Paulo, nos meses de janeiro a março, foi de $607 \mathrm{~mm}$ (Universidade Estadual Paulista Júlio de Mesquita Filho, 2011), o que gera dificuldades na aquisição de imagens ópticas de satélite livres de cobertura de nuvens.

O uso de dados provenientes de sensores de radar de abertura sintética (SAR, "synthetic aperture radar") para o monitoramento de culturas de cana-de-açúcar pode ser promissor, principalmente por sua capacidade de obter dados remotos independentemente das 
condições do tempo e por apresentar pulsos de radiação com diferentes polarizações, que podem potencializar a discriminação de alvos (McNairn \& Brisco, 2004; Paradella et al., 2005; Silva et al., 2009; Baghdadi et al., 2010).

Baghdadi et al. (2009) estudaram plantios de cana-de-açúcar da Ilha Reunião, a leste de Madagascar, e verificaram que dados de radar na banda $\mathrm{L}$ (comprimento de onda em torno de $23 \mathrm{~cm}$ ) e polarizações $\mathrm{HH}$ (emite e recebe a onda na polarização horizontal) e HV (emite a onda na polarização horizontal e recebe na vertical) apresentaram alta correlação com a altura da cana-de-açúcar $(0-150 \mathrm{~cm})$. Os autores observaram, ainda, forte correlação entre coeficientes de retroespalhamento na polarização $\mathrm{HH}\left(\sigma_{\mathrm{HH}}^{\circ}\right)$ e o índice de vegetação por diferença normalizada (NDVI), derivados de diferentes estágios de crescimento. $\mathrm{Na}$ fase de maturação, os valores de $\sigma^{\circ}$ apresentaram forte queda, em consequência da diminuição do conteúdo de água nas plantas.

Lin et al. (2009) estudaram a cultura de cana-de-açúcar da Província de Guangdong, China, e observaram que, na banda $\mathrm{C}$ (comprimento de onda em torno de $5 \mathrm{~cm}$ ), as polarizações $\mathrm{HH}$ e HV eram sensíveis aos componentes verticais e horizontais da planta, principalmente folhas e caules. Os autores observaram alta correlação entre o índice de área foliar (IAF) e o retroespalhamento, este último proveniente da razão de polarização $\mathrm{HV} / \mathrm{HH}$. Isso ocorreu porque a polarização HV normalmente apresenta valores baixos de retroespalhamento nos estágios iniciais do ciclo fenológico, que, no entanto, aumentam com o aparecimento de mais folhas. Com o crescimento das plantas, o IAF varia com o aumento das estruturas vertical (caule) e horizontal (folhas) das plantas. Este processo gera variações no retroespalhamento das polarizações $\mathrm{HH}$ e HV ao longo do ciclo da cultura. A variação é maior na polarização $\mathrm{HV}$, em função de sua maior sensibilidade ao aumento do retroespalhamento volumétrico no dossel da cultura. Dessa forma, esses autores frisaram a importância de se analisarem imagens relacionadas às fases de plantio e de maturação. Nessas fases, o retroespalhamento da cultura da cana-de-açúcar (7 a $11 \mathrm{~dB})$ tende a ser bem mais distinto do retroespalhamento dos alvos adjacentes, com valores inferiores a $4 \mathrm{~dB}$.

Outros estudos têm enfatizado a utilização da banda L, em razão de sua elevada relação com a biomassa da planta (Dabrowska-Zielinska et al., 2007; Baghdadi et al., 2009; Joseph et al., 2010). Paloscia (1998), ao comparar valores de $\sigma^{\circ}$ das bandas C, L e P (comprimento de onda em torno de $70 \mathrm{~cm}$ ), para estimar IAF de trigo, milho e alfafa, concluiu que, na banda L, há maior quantidade de informação, principalmente na polarização HV.

O objetivo deste trabalho foi avaliar o potencial das imagens multipolarizadas do sensor-radar Palsar/Alos em diferenciar as fases fenológicas da cana-de-açúcar.

\section{Material e Métodos}

Aárea de estudo abrangeu lavouras de cana-de-açúcar na região nordeste do Estado de São Paulo, delimitada pelas coordenadas $20^{\circ} 46^{\prime} \mathrm{Se} 21^{\circ} 50^{\prime} \mathrm{Se} 47^{\circ} 16^{\prime} \mathrm{We} 48^{\circ} 14^{\prime} \mathrm{W}$. A região tem clima tipicamente tropical, e os meses de janeiro a março são chuvosos, com precipitação pluvial média de 607 mm, entre 1971 e 2000; os meses de janeiro e fevereiro são os mais quentes, com temperatura média de $24,3^{\circ} \mathrm{C}$; os meses de julho a setembro são os mais secos, com precipitação pluvial média de $118,2 \mathrm{~mm}$; e o mês de junho é o mês mais frio, com temperatura média de $18,6^{\circ} \mathrm{C}$ (Universidade Estadual Paulista Júlio de Mesquita Filho, 2011). A altitude da região varia de 500 a $800 \mathrm{~m}$, e a declividade varia de 0 a $8 \%$, com relevo plano a suavemente ondulado (Oliveira et al., 1999). Os Latossolos predominam na área (Martorano et al., 1999).

Os dados de campo e os mapas com a localização dos talhões foram cedidos pela usina proprietária da área de estudo (Figura 1). A usina possui 24 variedades de cana-de-açúcar plantadas em toda sua extensão. Porém, foram selecionadas três variedades que pudessem ser monitoradas, por estarem em estágios fenológicos diferentes: RB85-5156, seis talhões, RB86-7515, dez talhões; e RB92-5345, dez talhões.

De acordo com Hoffmann et al. (2008), a variedade RB85-5156 apresenta elevado entouceiramento principalmente na soca -, com colmos eretos, mas decumbentes na fase adulta, diâmetro fino a médio, cor verde-clara, presença de rachaduras e muita palha. Apresenta também ótima capacidade de brotação de soqueiras e alta precocidade. A variedade RB86-7515 possui alta velocidade de crescimento, porte alto, hábito de crescimento ereto, alta densidade de colmo, cor verde arroxeado, que se acentua quando a planta fica exposta, e fácil despalha. Esta variedade é tolerante à seca, tem boa brotação de soqueira mesmo quando colhida crua, alto teor de sacarose, crescimento rápido e alta produtividade. A variedade RB92-5345 caracteriza-se pelo desenvolvimento rápido, hábito de crescimento ereto, difícil despalha, diâmetro de colmo médio, coloração roxo-amarelada quando exposta ao 
sol, presença destacada de cera, e gema ovalada. Possui também alto teor de sacarose, alta produtividade e alto teor de fibra no início de safra.

Os valores de $\sigma^{\circ}$ referentes foram analisados pelo cálculo da média do talhão, de acordo com a fase fenológica da variedade e estágio de corte. As três variedades foram divididas em: precoce, RB85-5156 colhida no início da safra, em abril-maio; média, RB86-7515 colhida na metade da safra, em junhoagosto; e tardia, RB92-5345 colhida no final da safra, em setembro-dezembro.

Para avaliar o potencial do sensor Palsar em discriminar as diferentes fases fenológicas ao longo de um ano, foram feitas duas análises: na primeira, para cada uma das imagens do sensor (quatro imagens no total), foram comparados os valores de retroespalhamento das três variedades entre si, que estavam em fases diferentes de crescimento na data específica da imagem; na segunda, foi realizada a comparação dos valores das fases de crescimento da mesma variedade ao longo do ano, uma variedade de cada vez.

As características das imagens do sensor Palsar foram pré-processadas pelas técnicas de correção radiométrica e geométrica (Tabela 1). A correção radiométrica envolveu a conversão dos níveis digitais em 16 bits e

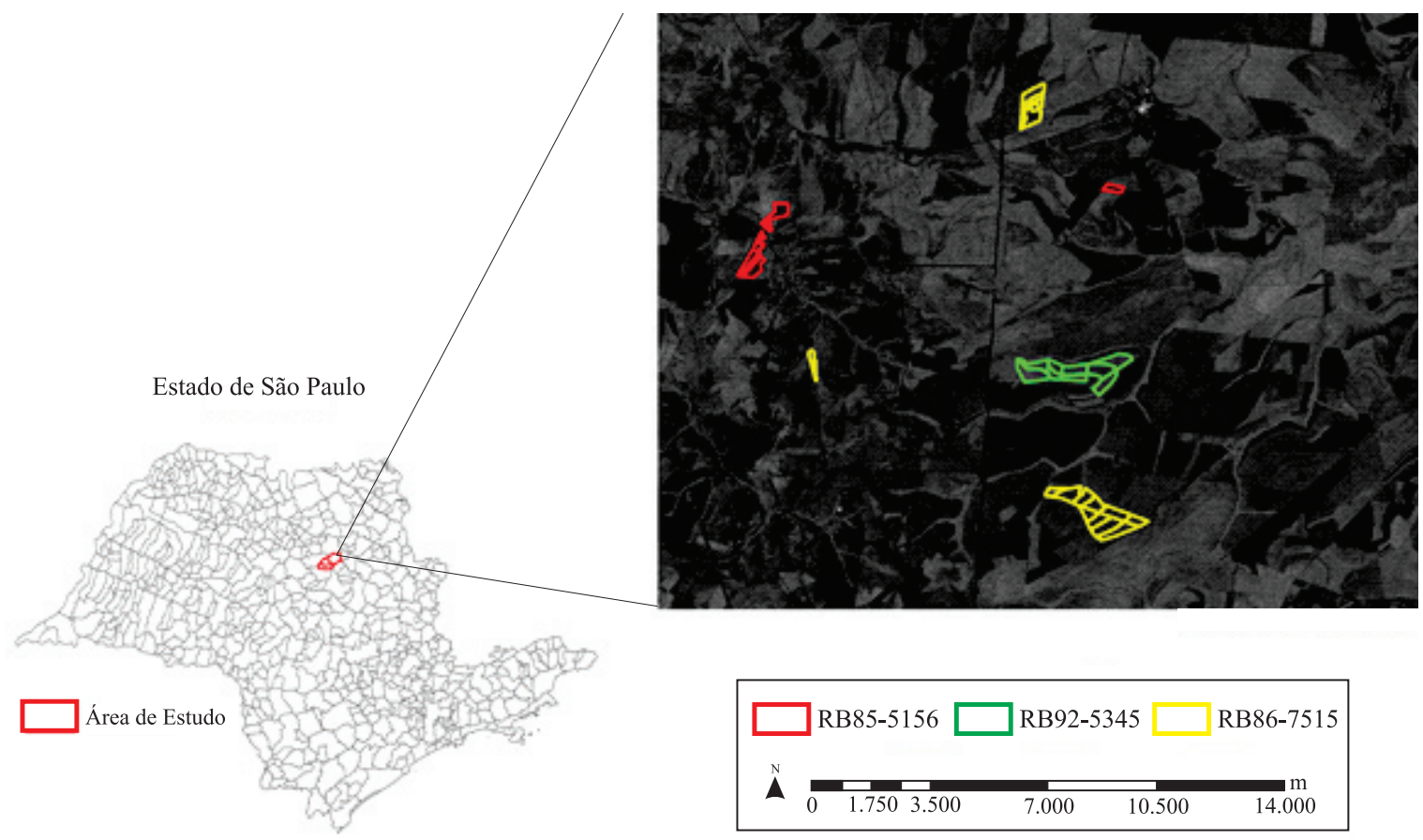

Figura 1. Distribuição dos talhões cultivados com as diferentes variedades de cana-de-açúcar - RB85 5156, RB92-5345 e

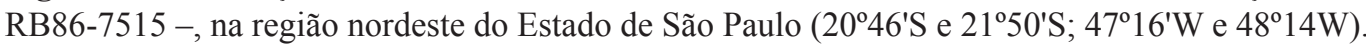

Tabela 1. Características das imagens do sensor Palsar.

\begin{tabular}{lccccc}
\hline Data da imagem & Polarização & Ângulo de incidência & Resolução espacial $(\mathrm{m})$ & Modo de imageamento & Número de visadas \\
\hline $22 / 2 / 2008$ & $\mathrm{HH}$ & $38^{\circ}$ & 6,25 & $\mathrm{FBS}^{(3)}$ & \\
$24 / 5 / 2008$ & $\mathrm{HH}+\mathrm{HV}$ & $38^{\circ}$ & 12,50 & $\mathrm{FBD}^{(4)}$ & 2 \\
$24 / 8 / 2008$ & $\mathrm{HH}+\mathrm{HV}$ & $38^{\circ}$ & 12,50 & $\mathrm{FBD}$ & 4 \\
$9 / 10 / 2008$ & $\mathrm{HH}+\mathrm{HV}$ & $38^{\circ}$ & 12,50 & $\mathrm{FBD}$ & 4 \\
\hline
\end{tabular}

HH, emite e recebe a onda na polarização horizontal; HV, emite a onda na polarização horizontal e recebe na vertical; FBS, imageamento fino, polarização simples; FBD, imageamento fino, polarização dupla. 
em amplitude para $\sigma^{\circ}$. Para isto, foi utilizada a equação $\sigma^{\circ}=10 \log \left(\mathrm{DN}^{2}\right)+\mathrm{CF}$ (Shimada et al., 2009), em que: DN é o valor digital correspondente ao valor do pixel da imagem com dados em amplitude; e CF é o fator de correção, cujos valores podem ser encontrados em Japan Aerospace Exploration Agency (2009). O valor do $\mathrm{CF}$ correspondeu a $-83 \mathrm{~dB}$, tanto para imagens $\mathrm{HH}$ nos modos "fine beam single" (FBS) e "fine beam dual" (FBD), quanto para imagens HV no modo FBD. Esta calibração permite compreender melhor os processos de retroespalhamento dos alvos e comparar imagens de datas e passagens distintas (Henderson \& Lewis, 1998).

A correção geométrica das imagens Palsar foi realizada com base na imagem ortorretificada (geocover) do Landsat ETM+ de 23 de março de 2001, disponível no endereço eletrônico da Universidade de Maryland, EUA, (Global Land Cover Facility, 2012), e projetada para o sistema de projeção cartográfica UTM (Universal Transversa de Mercator), datum WGS84 e fuso $23 \mathrm{~S}$.

Para verificar se houve diferenças nos valores de $\sigma^{\circ}$ da cana-de-açúcar, nos diferentes estágios de cortes, foi calculado o teste $\mathrm{z}$, com $5 \%$ de probabilidade. Para melhor entendimento do comportamento temporal de $\sigma^{\circ}$, foi calculado e analisado o balanço hídrico, pelo método de Thornthwaite \& Mather, com capacidade de água disponível (CAD) de $100 \mathrm{~mm}$. A evapotranspiração potencial (ETP) foi estimada pelo método de Thornthwaite.

Foi utilizado também o "standardized precipitation index" (SPI) cedido pelo Instituto Agronômico de Campinas (IAC). Dados de precipitação da área de estudo foram obtidos de 11 pluviômetros espalhados pela usina. O SPI é um indicador de seca, padronizado no espaço e no tempo, e baseia-se em séries históricas de dados, ao contrário da precipitação pluvial, que leva em consideração dados pontuais. As duas medidas são importantes, pois, enquanto o SPI indica a tendência dos dados de chuva e possibilita o monitoramento de secas, os dados de precipitação pluvial permitem mensurar a quantidade de chuva mais próxima da realidade.

Com a finalidade de verificar diferenças estatísticas nos valores médios de $\sigma^{\circ}$ provenientes de talhões de cana-de-açúcar em diferentes fases fenológicas ao longo do ciclo vegetativo, foi feita a análise descritiva dos dados e aplicados os testes de Mann-Whitney e de correlação de Spearman. Optou-se por utilizar o coeficiente de correlação de Spearman em razão do pequeno número de talhões. Das imagens FBD, foram geradas duas imagens-razão (HH/HV e HV/ $\mathrm{HH})$, que fizeram parte das análises. Valores de $\sigma^{\circ}$ de outros alvos, como mata, solo exposto e solo coberto por palha seca, também foram analisados por fazerem parte do processo de manejo da cultura em estudo.

\section{Resultados e Discussão}

Não houve precipitação pluvial nas datas de coleta das imagens Palsar, com exceção de 22 de fevereiro, quando houve chuva com intensidade de $4 \mathrm{~mm}$; tendo voltado a chover somente após o primeiro decêndio de outubro. As condições meteorológicas da área de estudo, nos dois primeiros meses de 2008, foram mais úmidas, acima das normais climatológicas, conforme indicado pelo valor positivo do SPI (Figura 2). Em março, foi observada uma condição ligeiramente mais seca. Nesse ano, a condição de seca, característica dos meses de maio a setembro para a referida localidade, foi marcante.

No mês de outubro e, especialmente, em novembro, verificaram-se valores negativos de SPI, o que indica quantidade de chuva abaixo da normal e deficiência
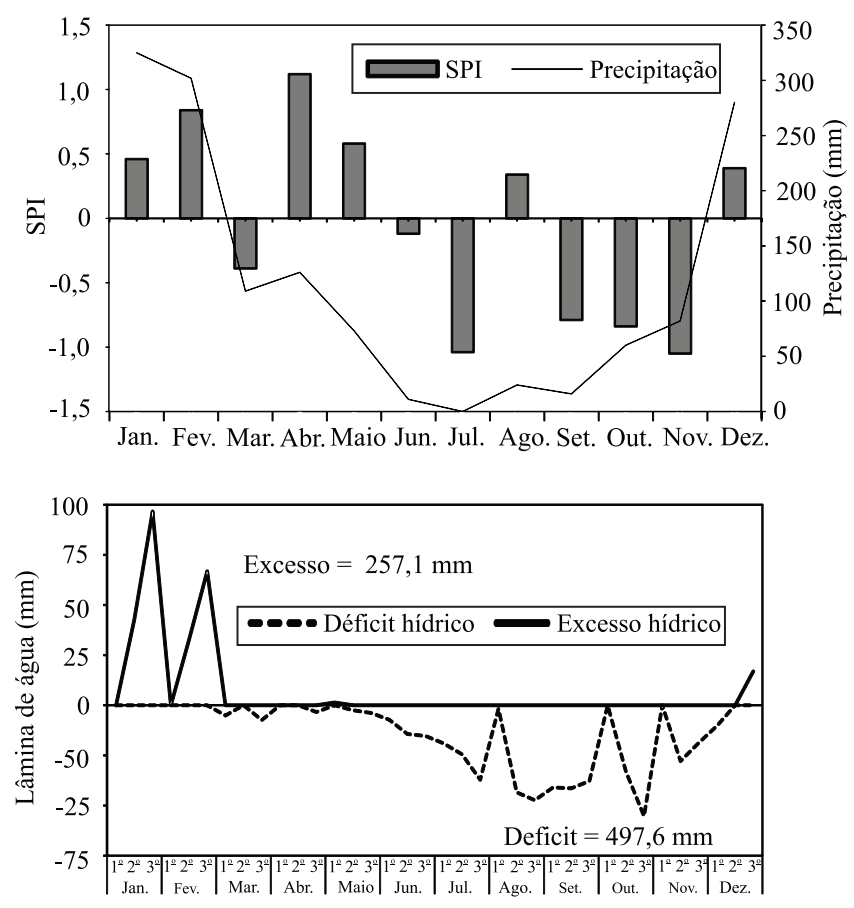

Figura 2. Valores de: A, precipitação e "standardized precipitation index" (SPI); e B, balanço hídrico decendial na área de estudo, no ano de 2008. 
hídrica. Portanto, houve atraso na retomada da estação chuvosa, que deveria ocorrer em outubro/novembro, e consequente atraso no desenvolvimento da cultura. Em dezembro, observou-se uma condição de mais umidade com o início das chuvas.

Para verificar se havia diferença no valor médio de $\sigma^{\circ}$ referente à cana-de-açúcar nos diferentes estágios de cortes, utilizou-se o teste $\mathrm{z}$ para as datas de fevereiro e maio, meses em que a maioria dos talhões ainda se encontrava com cana-de-açúcar plantada. A imagem de agosto não foi utilizada, pois somente continha nove dados (talhões de cana-de-açúcar), o que inviabilizou as análises. Para a imagem de 22 de fevereiro de 2008, o valor de $\mathrm{p}$ foi igual a 0,60 e, para a de 24 de maio, foi de 0,80 . Isto indica que não houve diferença significativa entre as médias dos valores de retroespalhamento da cana-de-açúcar do terceiro e quarto cortes. Portanto, não foi possível separar a cana-de-açúcar por estágio de corte.

Arelação entre $\sigma^{\circ}$ da cana-de-açúcar com polarização $\mathrm{HH}$ e HV é apresentada na Figura 3. Verificouse que a variedade RB92-5345, que estava com aproximadamente 12 meses de idade, ou seja, na fase de maturação, mostra alta correlação $(p=0,976)$ entre as polarizações, enquanto as outras duas variedades

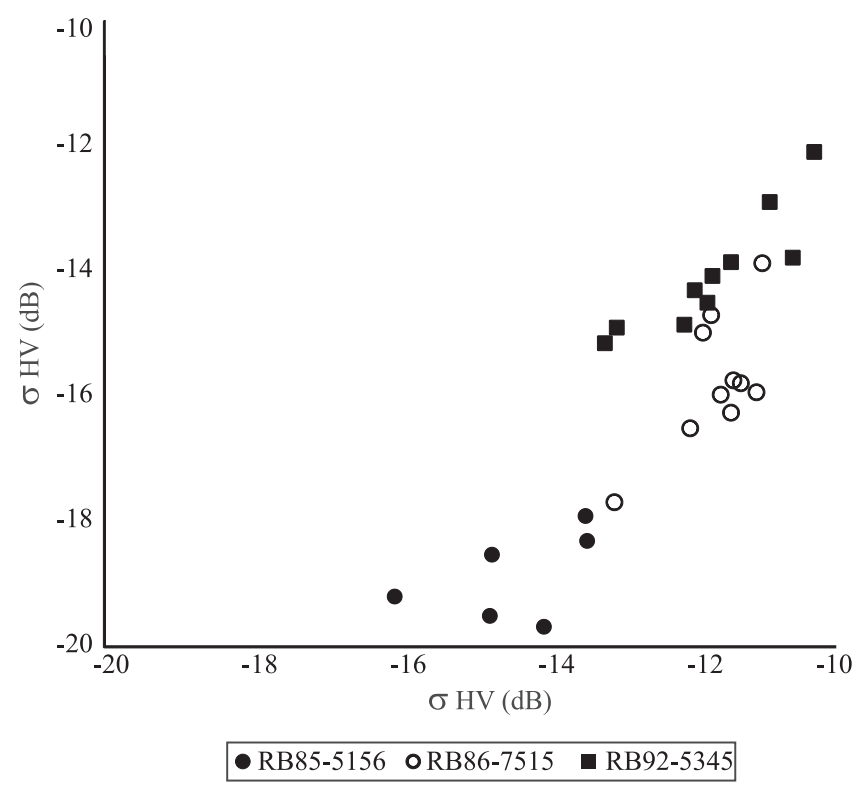

Figura 3. Retroespalhamento $\left(\sigma^{\circ}\right)$ nas polarizações $H H$ (emissão e recebimento de onda na polarização horizontal) e HV (emissão de onda na polarização horizontal e recebimento na vertical) de três variedades de cana de açúcar, em 24 de maio de 2008 . não apresentaram este comportamento: $p=0,333$, para RB86-7515 com seis a sete meses de idade, na fase de perfilhamento; e $p=0,543$, para RB85-5156 com um mês de idade, na fase de brotação e estabelecimento. Esse resultado pode indicar que as polarizações são complementares, conforme Evans et al. (1988), que apontaram as polarizações $\mathrm{HV}$ e $\mathrm{HH}$, como as que trazem melhor informação sobre o espalhamento volumétrico e superficial, respectivamente.

A variedade RB92-5345 apresentou alta correlação entre as polarizações, o que indica que houve redundância de informação. Uma das razões para isto é o fato de que os talhões dessa variedade estavam na fase de maturação. A fase de maturação caracteriza-se por menor quantidade de água interna na planta e queda de folhas. A desidratação nessa fase gera queda na constante dielétrica e, consequentemente, diminuição do $\sigma^{\circ}$ em ambas as polarizações.

Pode-se observar, também, que a polarização HV permitiu maior distinção entre os diferentes estádios de crescimento, exceto pelo equívoco entre alguns talhões de cana-de-açúcar das variedades RB86-7515 e RB92-5345 que, em razão de seus portes semelhantes (altura e folhas), apresentaram espalhamentos volumétricos semelhantes. Lin et al. (2009) também observaram que a polarização HV é mais sensível ao aumento do retroespalhamento volumétrico. No entanto, a polarização HH provocou maior confusão de respostas entre as fases fenológicas das variedades RB86-7515 (idade de sete meses) e RB92-5345 (idade de doze meses). Isso ocorreu pelo fato de as duas variedades estarem com alturas iguais (entre 3 e $3,5 \mathrm{~m}$ ) e terem dosséis com estruturas predominantemente eretas, tendo-se distinguido apenas a variedade RB85-5156, que estava com apenas um mês de idade.

Isto indica, novamente, que a polarização $\mathrm{HV}$ traz mais informações sobre a cultura (espalhamento volumétrico) do que a polarização $\mathrm{HH}$, o que concorda com as observações de Paloscia (1998) e Baghdadi et al (2010). Vale ressaltar que a utilização simultânea das polarizações HH e HV, na separação de fases fenológicas, foi bastante promissora.

Quando as variedades foram analisadas conjuntamente, observou-se, pelo comportamento espectral ao longo de um ano, nítida diferenciação entre as fases de crescimento. $\mathrm{Na}$ análise da imagem de 24 de maio de 2008, foi possível identificar os talhões da variedade RB85-5156, que já haviam sido colhidos, e facilmente 
diferenciá-los dos demais. Em razão da colheita, esses talhões apresentaram valores de retroespalhamento mais baixos do que os da variedade RB92-5345, que estava na fase de maturação, e RB86-7515, que estava no final da fase de crescimento dos colmos (Figura 4 A).

De acordo com o teste de Mann-Whitney para a polarização $\mathrm{HH}$ (Tabela 2), no mês de maio não
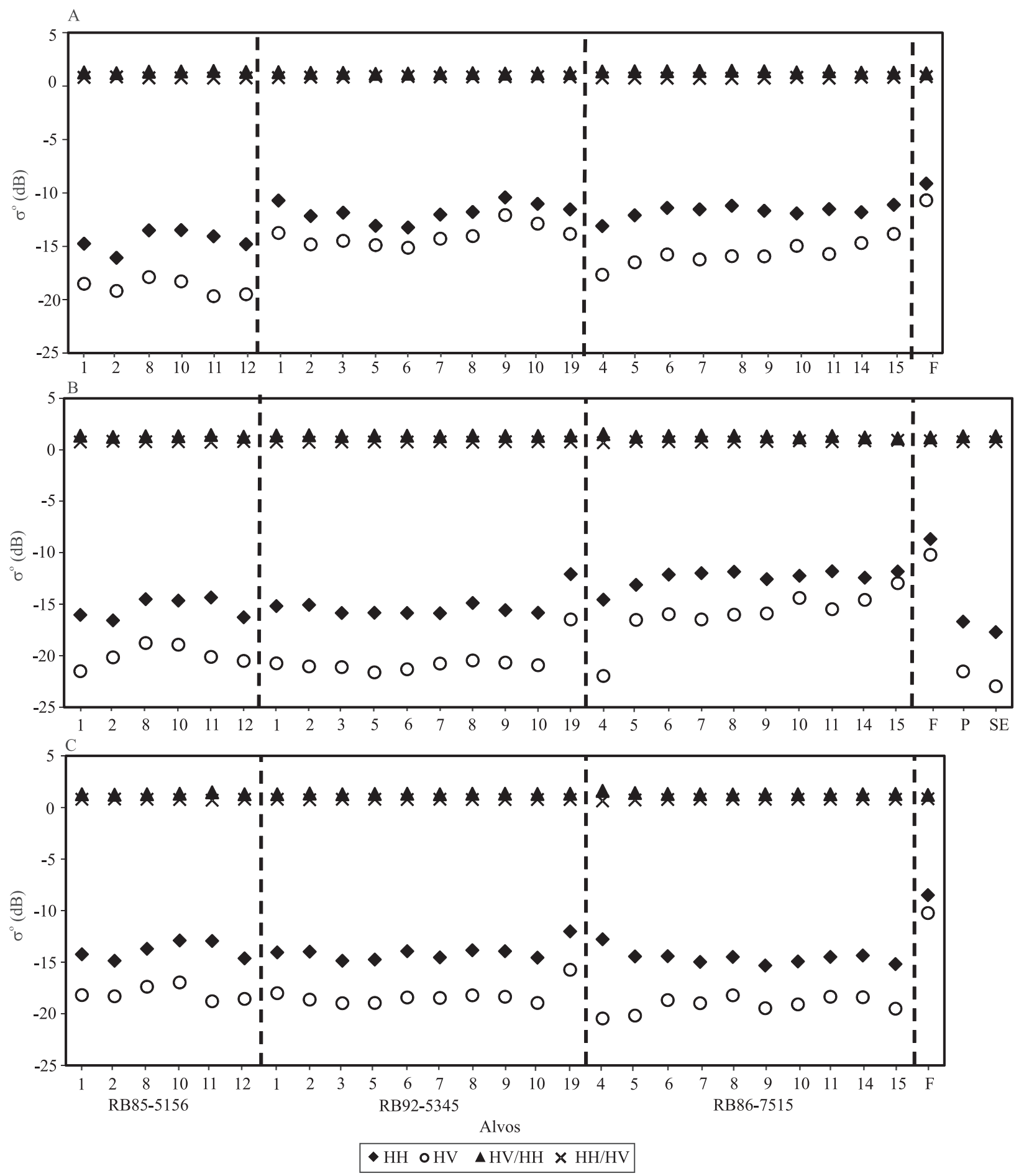

Figura 4. Variação dos valores de retroespalhamento $\left(\sigma^{\circ}\right)$, extraídos das imagens do sensor Palsar, das diferentes variedades de cana de açúcar, em diferentes fases de crescimento, e de diferentes alvos, nas seguintes datas de passagem: A, 24 de maio de 2008; B, 24 de agosto de 2008; e C, 9 de outubro de 2008. F, floresta; P, palha; SE, solo exposto. 
foi possível diferenciar as variedades RB92-5345 e RB86-7515, que estavam nas fases de maturação e de crescimento dos colmos, respectivamente. $\mathrm{Na}$ polarização $\mathrm{HV}$, essas duas variedades foram diferenciadas.

Nos dados de retroespalhamento da imagem de 24 de agosto de 2008, verificou-se que as variedades que já haviam sido colhidas, uma em abril/maio (RB85-5156) e a outra em junho (RB92-5345), apresentaram retroespalhamentos semelhantes para as polarizações HH e HV (Tabela 2). Como as duas variedades estavam no início da fase de perfilhamento e em período de deficiência hídrica, supõe-se que não havia água de chuva ou umidade de solo suficiente para influenciar os processos de restroespalhamento. A ocorrência de precipitação próxima à passagem do sensor altera as propriedades físicas e dielétricas da vegetação e do solo, o que causa homogeneização nos valores de retroespalhamento desses alvos (Lillesand et al., 2004). Assim, a única variável que poderia influenciar no retroespalhamento era o manejo (colheita com descarte da palha), que produziu rugosidade no solo cujo valor de $\sigma^{\circ}$ foi semelhante ao de superfícies com cana-de-açúcar. Essa rugosidade se manteve, em consequência do período de estiagem, desde a colheita até a data desta imagem (24 de agosto de 2008), em que os brotos apresentavam alturas inferiores a $30 \mathrm{~cm}$.

Ainda nos dados de retroespalhamento extraídos da imagem de 24 de agosto de 2008 (Figura 4 B), podese constatar a diferenciação das fases fenológicas das variedades RB85-5156 e RB92-5345, que estavam na fase de perfilhamento, e da variedade RB86-7515, que estava na fase de crescimento dos colmos, tanto na polarização $\mathrm{HH}$ quanto na $\mathrm{HV}$.

$\mathrm{O}$ valor do retroespalhamento do solo exposto (Figura 4B) foi menor do que o encontrado para a palha de cana-de-açúcar. Após a colheita mecânica, o solo ficou coberto pela palha da cana-de-açúcar em camada com cerca de $10 \mathrm{~cm}$ de altura. Depois de alguns dias (em torno de 7 dias), em uma nova operação, a palha da linha de plantio foi retirada e aleirada entre as linhas de plantio. Esta operação provocou uma sucessão de pequenos morros de palha, com aproximadamente 15 a $20 \mathrm{~cm}$ de altura, o que gerou aumento da rugosidade do terreno.

O fato de o solo exposto ter apresentado menores valores de $\sigma^{\circ}$, em comparação aos do talhão com palha, também pode ser explicado pelo manejo. A área ficou com solo exposto em duas situações: quando o talhão de cana-de-açúcar esteve em reforma ou quando a cana foi colhida manualmente. No período relativo a este trabalho, os valores de $\sigma^{\circ}$ corresponderam à primeira situação. A retirada das touceiras foi feita com grade niveladora, que, ao mesmo tempo em que nivelou o terreno, provocou seu destorroamento e diminuiu a rugosidade, tendo, consequentemente, diminuído o valor do retroespalhamento.

Para os dados de retroespalhamento extraídos da imagem de 9 de outubro de 2008, não houve diferenciação entre as fases fenológicas na polarização $\mathrm{HH}$ (Tabela 2). Tanto a variedade RB85-5156 quanto a RB92-5345 estavam na fase de perfilhamento e em condição de deficiência hídrica significativa (Figura 2 A), isto é, sem crescimento expressivo, tendose igualado em porte à RB86-7515, que foi colhida posteriormente (fase de brotação e estabelecimento), $o$ que determinou a similaridade no retroespalhamento. No mês de outubro, as três variedades se diferenciaram mais pela quantidade de perfilhos do que pela altura, e a variedade RB85-5156 apresentou o maior perfilhamento e a RB86-7515 o menor.

Porém, na polarização $\mathrm{HV}$, tanto a variedade RB85-5156 (cinco meses) quanto a RB92-5345 (quatro meses), que estavam na fase de perfilhamento,

Tabela 2. Teste de Mann-Whitney para diferentes variedades de cana de açúcar.

\begin{tabular}{|c|c|c|c|c|c|}
\hline \multirow[t]{2}{*}{ Comparação } & \multirow[t]{2}{*}{ Polarização } & \multicolumn{4}{|c|}{ Valor de $p$} \\
\hline & & $22 / 2 / 2008$ & $24 / 5 / 2008$ & $24 / 8 / 2008$ & $9 / 10 / 2008$ \\
\hline RB85-5156 vs. RB86 7515 & $\mathrm{HH}$ & $0,026^{*}$ & $0,001^{*}$ & $0,003 *$ & 0,143 \\
\hline RB85-5156 vs. RB92 5345 & $\mathrm{HH}$ & $0,003^{*}$ & $0,001^{*}$ & 0,786 & 0,745 \\
\hline RB86-7515 vs. RB92 5345 & $\mathrm{HH}$ & $0,000^{*}$ & 0,821 & $0,001^{*}$ & 0,121 \\
\hline RB85-5156 vs. RB86 7515 & $\mathrm{HV}$ & - & $0,001^{*}$ & $0,011^{*}$ & $0,020^{*}$ \\
\hline RB85-5156 vs. RB92 5345 & $\mathrm{HV}$ & - & $0,001^{*}$ & 0,116 & 0,303 \\
\hline RB86-7515 vs. RB92 5345 & $\mathrm{HV}$ & - & $0,003 *$ & $0,005^{*}$ & 0,050 \\
\hline
\end{tabular}

(1)Para a data 22/2/2008, não havia imagem com polarização HV. HH, emite e recebe a onda na polarização horizontal; HV, emite a onda na polarização horizontal e recebe na vertical.*Significativo a $5 \%$ de probabilidade. 
diferenciaram-se da variedade RB86-7515 (um mês), que estava na fase de brotação e estabelecimento. Como a polarização HV traz informações relacionadas ao espalhamento volumétrico, era de se esperar que plantas mais desenvolvidas se diferenciassem das menos desenvolvidas (Evans et al., 1988; Paloscia, 1998; Lin et al., 2009). Mais uma vez, os resultados comprovaram que a polarização $\mathrm{HV}$ traz mais informações sobre o alvo em questão.

Ao se analisar os valores de retroespalhamento da imagem de outros alvos, verificou-se que os valores de $\sigma^{\circ}$ referentes à floresta (Figura 4) se mantiveram constantes ao longo do ano, e que os valores da camada de palha da cana-de-açúcar após a colheita foram mais altos do que os do solo exposto, devido a diferenças no manejo. As razões $\sigma_{\mathrm{HV}}^{\circ} / \sigma_{\mathrm{HH}}^{\circ}$ e $\sigma_{\mathrm{HH}}^{\circ} / \sigma_{\mathrm{HV}}^{\circ}$ não trouxeram contribuições relevantes para este estudo, porque os valores de $\sigma_{\mathrm{HH}}^{\circ}$ e $\sigma_{\mathrm{HV}}^{\circ}$, nas imagens Palsar, apresentaram padrões similares. Quando houve um aumento no valor de $\sigma_{\mathrm{HH}}^{\circ}$, ocorreu também um aumento no valor de $\sigma_{\mathrm{HV}}^{\circ}$. Baghdadi et al. (2009) também não encontraram relação entre a razão $\sigma_{\mathrm{HH}}^{\circ} / \sigma_{\mathrm{HV}}^{\circ}$ das imagens Palsar e o estado da superfície das áreas plantadas com cana-de-açúcar. As fases fenológicas da cultura foram mascaradas, e não houve distinção entre a cana-de-açúcar colhida e a não colhida. Essas razões podem induzir a diagnósticos falhos, principalmente quando não se sabe se há realmente cana-de-açúcar plantada.

A variedade RB85-5156, por ser super precoce, foi colhida entre 17 de abril e 16 de maio de 2008 (Figura 5 A). Assim, em fevereiro, a cana-de-açúcar estava na fase de crescimento dos colmos, que se diferenciou significativamente das outras fases fenológicas observadas ao longo desse ano para a variedade. Em maio, o retroespalhamento foi controlado pela camada de palha que restou da cana-de-açúcar colhida. $\mathrm{O}$ aspecto rugoso, determinando pelo aleiramento da palha, gerou maior rugosidade na imagem do mês de maio, com tendência de aumento no retroespalhamento maior do que no mês de agosto.

Em agosto, a palha da variedade RB85-5156 já havia acamado e passou a ter uma espessura menor, em torno de $5 \mathrm{~cm}$, o que provocou suavização da superfície. Este fato causou aspecto diferente do encontrado no mês de maio, isto é, mesmo com o terreno em estado menos rugoso, o retroespalhamento ainda estava sob influência da palha. Nesse momento, a cultura estava no início da fase de perfilhamento, passava por um período de seca e, portanto, apresentava-se ainda muito pequena. Dessa forma, os valores de retroespalhamento foram mais baixos nessa data (24/8/2008), em comparação ao mês de maio.

Em outubro, a cana-de-açúcar teve um leve crescimento, e a contribuição da palha ao retroespalhamento da cultura diminuiu. Isto causou diferença significativa entre os valores de retroespalhamento da cana-de-açúcar, no mês de outubro (talhão com aproximadamente 5 meses, fim da fase de perfilhamento), e os valores de retroespalhamento no mês de agosto (talhão
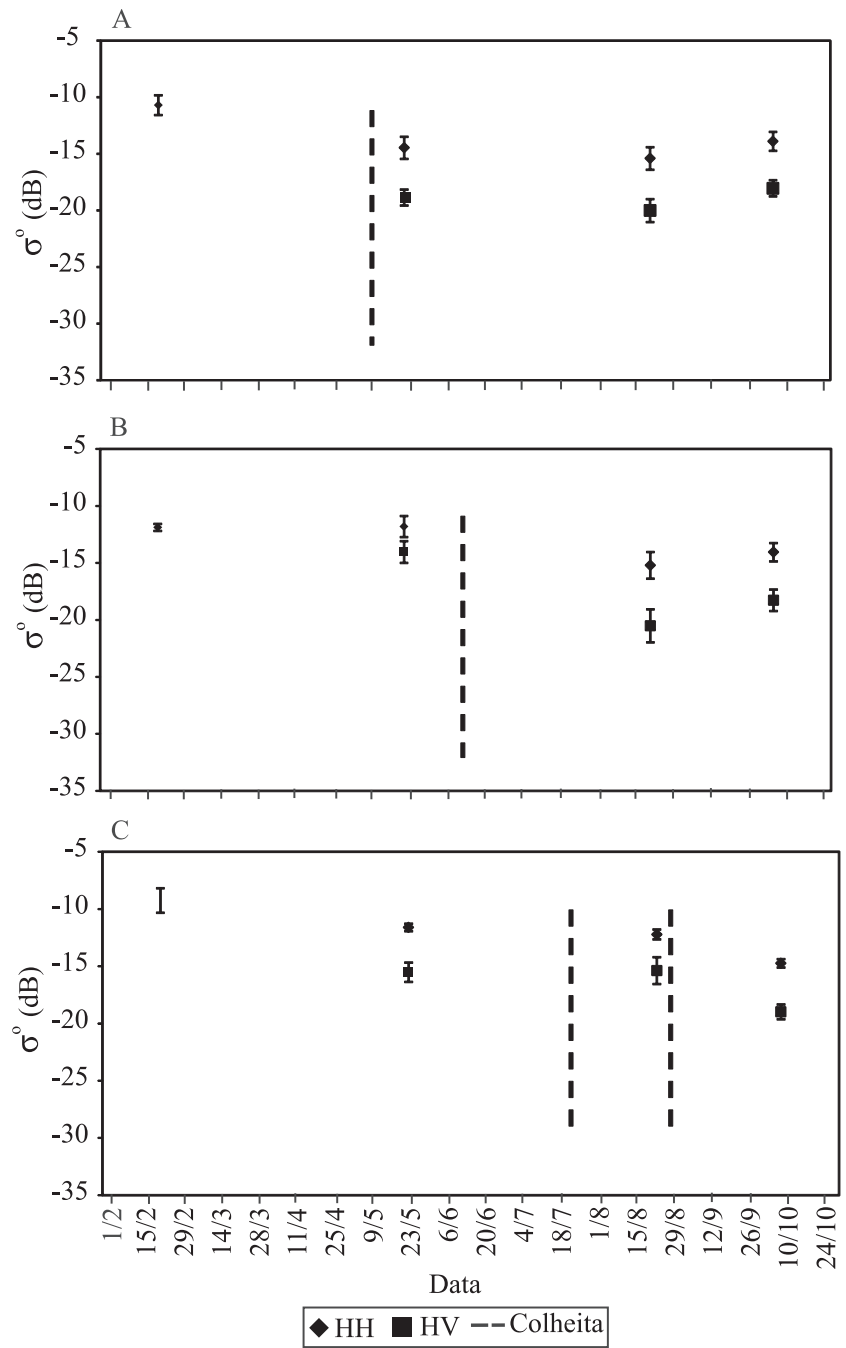

Figura 5. Comportamento temporal, quanto aos valores de retroespalhamento $\left(\sigma^{\circ}\right)$, das variedades de cana de açúcar RB85-5156 (A), RB92-5345 (B) e RB86-7515 (C), nas polarizações $\mathrm{HH}$ (emissão e recebimento de onda na polarização horizontal) e HV (emissão de onda na polarização horizontal e recebimento na vertical) no ano de 2008 . 
com aproximadamente 3 meses, início da fase de perfilhamento).

$\mathrm{Na}$ variedade RB92-5345, a colheita foi realizada entre 10 e 26 de junho. Assim, em fevereiro, a cana estava na fase de crescimento dos colmos e, em maio, na fase de maturação. Porém, não houve diferença significativa, a $5 \%$ de probabilidade, entre a fase de crescimento (9 meses) e de maturação (12 meses). Em agosto, após a colheita, a planta começou a rebrotar, mas com atraso, em razão da ocorrência de deficiência hídrica em julho e agosto. Na imagem de outubro, a cana estava maior e ainda na fase de perfilhamento, o que gerou um retroespalhamento mais alto (Figura 5 B), porém, não significativo em comparação ao mês de agosto.

A cana-de-açúcar da variedade RB86-7515 foi colhida entre 28 e 31 de agosto, com exceção de um único talhão que havia sido colhido em 27 de julho (Figura 5 C). Portanto, nas imagens de fevereiro, a cultura estava na fase de perfilhamento e, em maio, na fase de crescimento dos colmos. Foi observada diferença significativa entre essas duas fases de crescimento, o que corrobora o trabalho de Lin et al. (2009), que observaram que os valores de retroespalhamento, na banda $\mathrm{C}$, variam conforme a fase fenológica da cultura da cana-de-açúcar.

Em agosto, quando os talhões estavam na fase de maturação, houve queda nos valores de retroespalhamento, em consequência da diminuição do conteúdo de água nas plantas; o mesmo fato foi observado por Baghdadi et al. (2009). Porém, entre a fase de perfilhamento e a fase de maturação, assim como ocorreu na variedade RB92-5345, não houve diferença significativa. Por fim, em outubro, a cana estava no início da fase de perfilhamento, com aproximadamente $30 \mathrm{~cm}$ de altura, o que a diferenciou das demais fases fenológicas.

Para todas as variedades, o comportamento do retroespalhamento nas polarizações $\mathrm{HH}$ e $\mathrm{HV}$ seguiu a mesma tendência e foi válido para ambas as polarizações.

\section{Conclusões}

1. Os valores do retroespalhamento de imagens multipolarizadas, do sensor radar Palsar/Alos, mostram variações significativas entre as diferentes fases de crescimento da cana-de-açúcar.
2. A polarização HV (emissão de onda na polarização horizontal e recebimento na vertical) é mais eficiente do que a HH (emissão e recebimento de onda na polarização horizontal), na discriminação das diferentes fases de crescimento de cana-de-açúcar.

\section{Agradecimentos}

À Fundação de Amparo à Pesquisa do Estado de São Paulo, pelo apoio financeiro; ao Conselho Nacional de Desenvolvimento Científico e Tecnológico, pela concessão de bolsa; à Dra. Laura Hess, Universidade da Califórnia, pelo fornecimento das imagens Palsar/ Alos; e a Raízen, pelas sugestões e apoio de campo.

\section{Referências}

ABDEL-RAHMAN, E.M.; AHMED, F.B. The application of remote sensing techniques to sugarcane (Saccharum spp. hybrid) production: a review of the literature. International Journal of Remote Sensing, v.29, p.3753-3767, 2008.

BAGHDADI, N.; BOYER, N.; TODOROFF, P.; HAJJ, M.; BÉGUÉ, A. Potential of SAR sensors TerraSAR-X, ASAR/ ENVISAT and PALSAR/ALOS for monitoring sugarcane crops on Reunion Island. Remote Sensing of Environment, v.113, p.1724-1738, 2009.

BAGHDADI, N.; CRESSON, R.; TODOROFF, P.; MOINET, S. Multitemporal observations of sugarcane by TerraSAR-X images. Sensors, v.10, p.8899-8919, 2010.

DABROWSKA-ZIELINSKA, K.; INOUE, Y.; KOWALIK, W.; GRUSZCZYNSKA, M. Inferring the effect of plant and soil variables on C- and L-band SAR backscatter over agricultural fields, based on model analysis. Advances in Space Research, v.39, p.139-148, 2007.

EL HAJJ, M.; BÉGUÉ, A.; GUILLAUME, S.; MARTINÉ, J.F. Integrating SPOT-5 time series, crop growth modeling and expert knowledge for monitoring agricultural practices - the case of sugarcane harvest on Reunion Island. Remote Sensing of Environment, v. 113, p.2052-2061, 2009.

EVANS, D.L.; FARR, T.J.; VAN ZYL, J.J.; ZEBKER, H.A. Radar polarimetry: analysis tools and applications. IEEE Transactions on Geoscience and Remote Sensing, v.26, p.774-789, 1988.

GLOBAL LAND COVER FACILITY. Global Land Cover Facility: Earth Science Data Interface [home page]. Available at: $<$ http://glcfapp.glcf.umd.edu:8080/esdi/index.jsp >. Accessed on: 5 Sept. 2012

HENDERSON, F.M.; LEWIS, A.J. Principles and applications of imaging radar: manual of remote sensing. New York: John Wiley and Sons, 1998. 866p.

HOFFMANN, H.P.; SANTOS, E.G.D.; BASSINELLO, A.I.; VIEIRA, M.A.S. Variedades RB de cana-de-açúcar. Araras: Universidade Federal de São Carlos, 2008. 30p. 
JAPAN AEROSPACE EXPLORATION AGENCY. PALSAR calibration factor updated. Available at: $<$ https://auig.eoc.jaxa.jp/ auigs/en/doc/an/20090109en_3.html>. Accessed on: 8 June 2009.

JOSEPH, A.T.; VAN DER VELDE, R.; O'NEILL, P.E.; LANG, R.; GISH, T. Effects of corn on C- and L-band radar backscatter: a correction method for soil moisture retrieval. Remote Sensing of Environment, v.114, p.2417-2430, 2010.

LEBOURGEOIS, V.; CHOPART, J.-L.; BÉGUÉ, A.; LE MÉZO, L. Towards using a thermal infrared index combined with water balance modeling to monitor sugarcane irrigation in a tropical environment. Agricultural Water Management, v.97, p.75-82, 2010.

LILLESAND, T.M.; KIEFER, R.W.; CHIPMAN, J.W. Remote sensing and image interpretation. 5.ed. New York: John Wiley and Sons, 2004. 763p.

LIN, H.; CHEN, J.; PEI, Z.; ZHANG, S.; HU, X. Monitoring sugarcane growth using ENVISAT ASAR data. IEEE Transactions on Geoscience and Remote Sensing, v.47, p.2572-2580, 2009.

MARTORANO, L.G.; ANGELOCCI, L.R.; VETTORAZZI, C.A.; VALENTE, R. de O.A. Zoneamento agroecológico para a região de Ribeirão Preto utilizando um sistema de informações geográficas. Scientia Agricola, v.56, p.739-747, 1999.

MCNAIRN, H.; BRISCO, B. The application of C-band polarimetric SAR for agriculture: a review. Canadian Journal of Remote Sensing, v.30, p.525-542, 2004.

OLIVEIRA, J.B. de; CAMARGO, M.N.; ROSSI, M.; CALDERANO FILHO, B. Mapa pedológico do Estado de São Paulo: legenda expandida. Campinas: Instituto Agronômico; Rio de Janeiro: Embrapa Solos, 1999. 64p.

PALOSCIA, S. An empirical approach to estimating leaf area index from multifrequency SAR data. International Journal of Remote Sensing, v.19, p.361-366, 1998.
PARADELLA, W.R.; SANTOS, A.R. dos; VENEZIANI, P.; CUNHA, E.S.P. da. Radares imageadores nas Geociências: estado da arte e perspectivas. Revista Brasileira de Cartografia, n.57, p.56-62, 2005.

PICOLI, M.C.A.; RUDORFF, B.F.T.; RIZZI, R.; GIAROLLA, A. Índice de vegetação do sensor MODIS na estimativa da produtividade agrícola da cana-de-açúcar. Bragantia, v.68, p.789-795, 2009.

RUDORFF, B.F.T.; AGUIAR, D.A.; SILVA, W.F.; SUGAWARA, L.M.; ADAMI, M.; MOREIRA, M.A. Studies on the rapid expansion of sugarcane for ethanol production in São Paulo state (Brazil) using Landsat data. Remote Sensing, v.2, p.1057-1076, 2010.

SHIMADA, M.; ISOGUCHI, O.; TADONO, T.; ISONO, K. PALSAR radiometric and geometric calibration. IEEE Transactions on Geoscience and Remote Sensing, v.47, p.3915-3932, 2009.

SILVA, W.F.; RUDORFF, B.F.T.; FORMAGGIO, A.R.; PARADELLA, W.R.; MURA, J.C. Discrimination of agricultural crops in a tropical semi-arid region of Brazil based on L-band polarimetric airborne SAR data. ISPRS Journal of Photogrammetry and Remote Sensing, v.64, p.458-463, 2009.

UNIVERSIDADE ESTADUAL PAULISTA JÚLIO DE MESQUITA FILHO. Resenha meteorológica do período 1971 - 2000. Jaboticabal. Disponível em: <http://jaguar.fcav.unesp. br/portal_agromet/int_conteudo_sem_img.php?conteudo $=180>$. Acesso em: 20 jan. 2011.

VIEIRA, M.A.; FORMAGGIO, A.R.; RENNÓ, C.D.; ATZBERGER, C.; AGUIAR, D.A.; MELLO, M.P. Object-based image analysis and data mining applied to a remotely sensed Landsat time-series to map sugarcane over large areas. Remote Sensing of Environment, v.123, p.553-562, 2012.

$\overline{\text { Recebido em } 27 \text { de junho de } 2011 \text { e aprovado em } 29 \text { de agosto de } 2012}$ 